Supporting Information

\title{
Programmable Soft-Matter Electronics
}

Artemii S. Ivanov, ${ }^{1}$ Konstantin G. Nikolaev, ${ }^{1}$ Alexander S. Novikov, ${ }^{1}$ Stanislav O. Yurchenko, ${ }^{2}$ Kostya S. Novoselov, ${ }^{3}$ Daria V. Andreeva ${ }^{3}$ and Ekaterina V. Skorb ${ }^{1, *}$

${ }^{1}$ Infochemistry Scientific Center of ITMO University, 9, Lomonosova str., Saint Petersburg, 191002, Russia

${ }^{2}$ Bauman Moscow State Technical University, Moscow 105005, Russia

${ }^{3}$ Department of Materials Science and Engineering, National University of Singapore, Singapore117575, Singapore

\section{Corresponding Author}

skorb@itmo.ru

\section{Experimental Section}

Chemicals: Polyacrylic acid (PAA; molar mass $450000 \mathrm{~g} \mathrm{~mol}^{-1}$ ), polyethileneimine (PEI branched; molar mass $750000 \mathrm{~g}$ mol-1), agar (powder) are purchased from Sigma-Aldrich. Monopotassium phosphate $\left(\mathrm{KH}_{2} \mathrm{PO}_{4}\right)$ and disodium phosphate $\left(\mathrm{Na}_{2} \mathrm{HPO}_{4}\right)$ for the preparation of PBS are purchased from LenReactiv.

Gel preparation: $0.1 \mathrm{~g}$ of agar is added to $10 \mathrm{ml}$ of distilled water. Then, $1 \mathrm{wt} . \%$ PAA solution in the amount of $10 \mu \mathrm{l}$ and $5 \mu \mathrm{l}$ is added to obtain $\mathrm{pH} 4.8$ and 5.4, respectively. In the case of PEI, 
$10 \mu \mathrm{l}$ of $1 \mathrm{wt} . \%$ solution of PEI is added, and $\mathrm{pH}$ is adjusted by adding the required amount of 1 M hydrochloric acid. Next, the resulting solution is stirred, heated, and poured into a Petri dish for cooling. For buffered gels for solution preparation, we use $10 \mathrm{ml}$ of $0.07 \mathrm{M}$ phosphate buffer with $\mathrm{pH} 4.8$ and 5.4. 1 wt.\% PAA solution in the amount of $10 \mu \mathrm{l}$ and $5 \mu \mathrm{l}$ is added to the buffer with pH 4.8 and 5.4, respectively. In the case of PEI, $10 \mu \mathrm{l}$ of $1 \mathrm{wt} . \%$ solution of PEI is added to the buffer solution.

Instruments: Current-voltage (I-V) characteristic curves are measured by Keithley 6430. For database I-V curves are measured three times for three samples in the voltage sweep mode in the following potential values: -0.02 to $0.02 \mathrm{~V},-0.05$ to $0.05 \mathrm{~V},-0.1$ to $0.1 \mathrm{~V},-0.5$ to $0.5 \mathrm{~V},-1$ to $1 \mathrm{~V}$, -2 to $2 \mathrm{~V},-5$ to $5 \mathrm{~V}$. Then at least 5 times the ON-OFF mode was used with switching the application of negative and positive potential equal to $5 \mathrm{~V}$ for a duration of 1 second for 10 times. Then again, in the voltage sweep mode, three consecutive measurements were done in the range of -5 to $5 \mathrm{~V}$ : $(-5$ to $5 \mathrm{~V}) \mathrm{n}$.

Software: For machine learning calculations Weka v3.8.4 (Waikato Environment for Knowledge Analysis) is used. For data processing by supervised learning method, a multilayer perceptron model with the following parameters was used: learning rate -0.3 , momentum -0.2 , random seed -0 , the number of nodes in a hidden layer -12 . For unsupervised learning EM clustering model with the following parameters was used: maxiterations -100 , numFolds -10 , numKMeansRuns -10 , seed -100 .

DFT calculation: The quantum chemical calculations and full geometry optimization of all model structures were carried out with the help of the ORCA 4.2.1 program package. ${ }^{1}$ Convergence tolerances for geometry optimization procedure: energy change $=5.0000 \mathrm{e}-06 \mathrm{Eh}$, maximal gradient $=3.0000 \mathrm{e}-04 \mathrm{Eh} /$ Bohr, RMS gradient $=1.0000 \mathrm{e}-04 \mathrm{Eh} /$ Bohr, maximal 
displacement $=4.0000 \mathrm{e}-03 \mathrm{Bohr}, \mathrm{RMS}$ displacement $=2.0000 \mathrm{e}-03 \mathrm{Bohr}$. The TightSCF and Grid 4 specifications were used in all cases. Symmetry operations have not been applied during the geometry optimization procedure for all model structures. The Hessian matrices were calculated for all optimized model structures in order to prove the location of correct minima on the potential energy surfaces (no imaginary frequencies in all cases) and to estimate the thermodynamic parameters, the latter being calculated at $298.15 \mathrm{~K}$ and $1.00 \mathrm{~atm}$ (Table S1).

Table S1. Calculated Gibbs free energies change for reactions proceeding in a hydrogel.

\begin{tabular}{|c|c|c|}
\hline Reaction & $\begin{array}{c}\Delta \mathrm{G}, \\
\mathrm{kJ} / \mathrm{mol}\end{array}$ & Level of theory \\
\hline $\mathrm{PEI}+\mathrm{Ga}(\mathrm{OH})_{2}{ }^{+}=\mathrm{PEI} \cdots \mathrm{N}-\mathrm{Ga}(\mathrm{OH})_{2}{ }^{+}$ & -296.3 & $\omega \mathrm{B} 97 \mathrm{X}-\mathrm{D} 3 / 6-311++\mathrm{G}^{* *}$ \\
\hline $\mathrm{PEI}+\mathrm{Ga}(\mathrm{OH})_{2}{ }^{+}=\mathrm{PEI} \cdots \mathrm{NH}-\mathrm{Ga}(\mathrm{OH})_{2}{ }^{+}$ & -286.3 & $\omega \mathrm{B} 97 \mathrm{X}-\mathrm{D} 3 / 6-311++\mathrm{G}^{* *}$ \\
\hline $\mathrm{PEI}+\mathrm{Ga}(\mathrm{OH})_{2}{ }^{+}=\mathrm{PEI} \cdots \mathrm{NH}_{2}-\mathrm{Ga}(\mathrm{OH})_{2}{ }^{+}$ & -282.2 & $\omega \mathrm{B} 97 \mathrm{X}-\mathrm{D} 3 / 6-311++\mathrm{G}^{* *}$ \\
\hline $\mathrm{PAA}+\mathrm{Ga}(\mathrm{OH})_{2}{ }^{+}=\mathrm{PAA} \cdots \mathrm{Ga}(\mathrm{OH})_{2}{ }^{+}$ & -225.9 & $\omega \mathrm{B} 97 \mathrm{X}-\mathrm{D} 3 / 6-311++\mathrm{G}^{* *}$ \\
\hline $\mathrm{PEI}+\mathrm{Ga}(\mathrm{OH})_{2}{ }^{+}=\mathrm{PEI} \cdots \mathrm{N}-\mathrm{Ga}(\mathrm{OH})_{2}{ }^{+}$ & -261.2 & B3LYP/aug-cc-pVDZ \\
\hline $\mathrm{PEI}+\mathrm{Ga}(\mathrm{OH})_{2}{ }^{+}=\mathrm{PEI} \cdots \mathrm{NH}-\mathrm{Ga}(\mathrm{OH})_{2}{ }^{+}$ & -257.2 & B3LYP/aug-cc-pVDZ \\
\hline $\mathrm{PEI}+\mathrm{Ga}(\mathrm{OH})_{2}{ }^{+}=\mathrm{PEI} \cdots \mathrm{NH}{ }_{2}-\mathrm{Ga}(\mathrm{OH})_{2}{ }^{+}$ & -258.9 & B3LYP/aug-cc-pVDZ \\
\hline $\mathrm{PAA}+\mathrm{Ga}(\mathrm{OH})_{2}{ }^{+}=\mathrm{PAA} \cdots \mathrm{Ga}(\mathrm{OH})_{2}{ }^{+}$ & -209.2 & B3LYP/aug-cc-pVDZ \\
\hline
\end{tabular}


Table S2. Cartesian atomic coordinates for all optimized model structures.

\begin{tabular}{|c|c|c|c|}
\hline Atom & $\mathrm{X}$ & $\mathrm{Y}$ & $\mathrm{Z}$ \\
\hline \multicolumn{4}{|c|}{$\mathrm{Ga}(\mathrm{OH})_{2}^{+}$} \\
\hline $\mathrm{Ga}$ & -2.08437921366373 & 1.09270357213407 & -0.58649777243373 \\
\hline $\mathrm{O}$ & -0.71663390583413 & 0.29036313230377 & 0.01441382059249 \\
\hline $\mathrm{O}$ & -3.60732634644608 & 1.72930219239553 & -0.97498269156269 \\
\hline $\mathrm{H}$ & -3.88677742020728 & 1.88573147152935 & -1.88598608195452 \\
\hline $\mathrm{H}$ & 0.12553688615124 & 0.73966963163728 & 0.16053272535845 \\
\hline \multicolumn{4}{|l|}{ PAA } \\
\hline $\mathrm{C}$ & -8.89597812059932 & 3.25970890975821 & -2.24465520187024 \\
\hline $\mathrm{O}$ & -9.81040944416837 & 3.00916141856178 & -2.97543206224127 \\
\hline $\mathrm{C}$ & -7.53827167985337 & 2.57121133326856 & -2.33440353019326 \\
\hline $\mathrm{O}$ & -9.03548847324037 & 4.20964348787954 & -1.29218174305102 \\
\hline $\mathrm{H}$ & -8.21742017159981 & 4.29982098995611 & -0.79744891594990 \\
\hline $\mathrm{C}$ & -6.84592012746449 & 2.99893057359009 & -3.63722490455395 \\
\hline $\mathrm{C}$ & -7.73011113445174 & 1.05549238132786 & -2.26706342937501 \\
\hline
\end{tabular}




\begin{tabular}{|c|c|c|c|}
\hline $\mathrm{H}$ & -6.90904426623721 & 2.88818590197352 & -1.49049347993698 \\
\hline $\mathrm{H}$ & -7.47232269924896 & 2.67751244358201 & -4.47582155431464 \\
\hline $\mathrm{H}$ & -5.90355314806983 & 2.44605348238877 & -3.71431144354545 \\
\hline $\mathrm{C}$ & -6.57870349316875 & 4.49850861134593 & -3.72845217560074 \\
\hline $\mathrm{H}$ & -8.37217259720960 & 0.72407502252410 & -3.08595573066848 \\
\hline $\mathrm{H}$ & -8.19630447401429 & 0.74922368656400 & -1.32721382553604 \\
\hline $\mathrm{H}$ & -6.76573635304476 & 0.54810287813532 & -2.35126500591215 \\
\hline $\mathrm{H}$ & -7.50882688027942 & 5.07496359966377 & -3.71951301155672 \\
\hline $\mathrm{H}$ & -6.05117551772711 & 4.74591926670758 & -4.65235152543939 \\
\hline $\mathrm{H}$ & -5.95913141962246 & 4.84378601277282 & -2.89350246025471 \\
\hline \multicolumn{4}{|c|}{$\mathrm{PAA} \cdots \mathrm{Ga}(\mathrm{OH})_{2}{ }^{+}$} \\
\hline $\mathrm{C}$ & -8.65861121432188 & 3.56191293149404 & -1.86626360755915 \\
\hline $\mathrm{O}$ & -9.80724096229172 & 3.31581156412900 & -2.28340651885493 \\
\hline $\mathrm{C}$ & -7.50686952122474 & 2.67544703962759 & -2.20365974196555 \\
\hline $\mathrm{O}$ & -8.51901473585849 & 4.64769170573110 & -1.15874630139158 \\
\hline $\mathrm{H}$ & -7.60664573285370 & 4.80142592530818 & -0.87946256703681 \\
\hline
\end{tabular}




\begin{tabular}{|c|c|c|c|}
\hline $\mathrm{C}$ & -7.12248514521985 & 2.94847162665666 & -3.67985661899600 \\
\hline $\mathrm{C}$ & -7.86292488825786 & 1.20538470577102 & -1.95902534143088 \\
\hline $\mathrm{H}$ & -6.66341207326095 & 2.96459976691438 & -1.56494377177009 \\
\hline $\mathrm{H}$ & -7.94293005620362 & 2.60788167552225 & -4.31892678376818 \\
\hline $\mathrm{H}$ & -6.26775270415413 & 2.30200181480384 & -3.89444965169357 \\
\hline $\mathrm{C}$ & -6.77414152192150 & 4.40244747414031 & -3.98207372368627 \\
\hline $\mathrm{H}$ & -8.70135312306623 & 0.90017653055271 & -2.58720133161549 \\
\hline $\mathrm{H}$ & -8.12111826285311 & 1.01620048312474 & -0.91525152439346 \\
\hline $\mathrm{H}$ & -7.00066573396074 & 0.58690126697791 & -2.21159012077261 \\
\hline $\mathrm{H}$ & -7.63666522278883 & 5.06999921419415 & -3.87812135196124 \\
\hline $\mathrm{H}$ & -6.42381791510295 & 4.50143144158605 & -5.01023869606744 \\
\hline $\mathrm{H}$ & -5.97164336322410 & 4.76860395715202 & -3.33308189254768 \\
\hline $\mathrm{Ga}$ & -11.32867901116149 & 4.44539680298985 & -2.20365689273137 \\
\hline $\mathrm{O}$ & -12.41092834066101 & 3.76082103834681 & -1.02813100717024 \\
\hline $\mathrm{H}$ & -13.27333021713350 & 4.15791717486494 & -0.88432695141199 \\
\hline $\mathrm{O}$ & -11.01263736236825 & 5.68318506513351 & -3.38959084911078 \\
\hline
\end{tabular}




\begin{tabular}{|c|c|c|c|}
\hline $\mathrm{H}$ & -11.67769289211054 & 6.35012079497858 & -3.57594475406444 \\
\hline \multicolumn{4}{|c|}{ PEI } \\
\hline $\mathrm{C}$ & -3.57664216735730 & 2.51242830541970 & 0.75725017980698 \\
\hline $\mathrm{C}$ & -2.57670308743881 & 3.29922564934866 & -0.08378779608750 \\
\hline $\mathrm{H}$ & -4.29568891768772 & 1.97871655750607 & 0.12582066772616 \\
\hline $\mathrm{H}$ & -4.14459238616855 & 3.17234845545611 & 1.41821365601944 \\
\hline $\mathrm{H}$ & -3.06221633807092 & 1.77752201349895 & 1.38259819635467 \\
\hline $\mathrm{C}$ & -1.75589539153252 & 2.43355835629577 & -1.04015122499209 \\
\hline $\mathrm{H}$ & -1.88331764705078 & 3.83092998386569 & 0.57767736044222 \\
\hline $\mathrm{H}$ & -3.10368857626349 & 4.06159067806767 & -0.67092761997505 \\
\hline $\mathrm{N}$ & -0.93891025145756 & 1.43049735485257 & -0.35713666387229 \\
\hline $\mathrm{H}$ & -1.10178755960649 & 3.08754838183115 & -1.62791862932669 \\
\hline $\mathrm{H}$ & -2.42541042310094 & 1.94896402162547 & -1.76821039077316 \\
\hline $\mathrm{C}$ & 0.47018334153559 & 1.48878407624437 & -0.71248463389005 \\
\hline $\mathrm{C}$ & 1.18723676673392 & 2.64259351785584 & -0.02435008805611 \\
\hline $\mathrm{H}$ & 0.60630166092478 & 1.56340450457662 & -1.80913870581896 \\
\hline
\end{tabular}




\begin{tabular}{|c|c|c|c|}
\hline $\mathrm{H}$ & 0.95847616351266 & 0.56076479321748 & -0.40633157374570 \\
\hline $\mathrm{N}$ & 2.58217549499024 & 2.69598431541444 & -0.42701579626642 \\
\hline $\mathrm{H}$ & 1.13997967026466 & 2.48084574378939 & 1.05809953958382 \\
\hline $\mathrm{H}$ & 0.65508176852253 & 3.59157055278590 & -0.22096904012543 \\
\hline $\mathrm{C}$ & 3.32543714886754 & 3.76018977596783 & 0.22406560200617 \\
\hline $\mathrm{H}$ & 2.87881746472531 & 4.76034277239092 & 0.09138521804909 \\
\hline $\mathrm{H}$ & 4.34804204407324 & 3.78544161158050 & -0.15882096713347 \\
\hline $\mathrm{H}$ & 3.38056576098171 & 3.56114022612699 & 1.29850221126612 \\
\hline $\mathrm{H}$ & 2.64287077263489 & 2.80015106724971 & -1.43318643092263 \\
\hline $\mathrm{C}$ & -1.48360846606667 & 0.08059434405668 & -0.41509887596325 \\
\hline $\mathrm{C}$ & -1.12597605603732 & -0.74465123886536 & 0.81673494265260 \\
\hline $\mathrm{H}$ & -1.16275811222285 & -0.46400783948788 & -1.31975447580995 \\
\hline $\mathrm{H}$ & -2.57438050425692 & 0.15480923901210 & -0.46937096401182 \\
\hline $\mathrm{N}$ & -1.70729692208795 & -2.07991437023378 & 0.70638145239234 \\
\hline $\mathrm{H}$ & -1.44445488961360 & -0.18555933680255 & 1.70934408328369 \\
\hline $\mathrm{H}$ & -0.03946995503616 & -0.85607267367579 & 0.88516837413104 \\
\hline
\end{tabular}




\begin{tabular}{|c|c|c|c|}
\hline $\mathrm{H}$ & -2.71593677592893 & -2.04306419750470 & 0.79718390143716 \\
\hline $\mathrm{H}$ & -1.36382363078154 & -2.69061664146652 & 1.43622849161908 \\
\hline \multicolumn{4}{|c|}{ PEI $\cdots \mathrm{N}-\mathrm{Ga}(\mathrm{OH})_{2}{ }^{+}$} \\
\hline $\mathrm{C}$ & -2.31252975167028 & 3.37660403551364 & -3.37479760514531 \\
\hline $\mathrm{C}$ & -1.55360312050348 & 2.24730058136459 & -2.67941885806152 \\
\hline $\mathrm{C}$ & -1.69135111376480 & 2.37748997344654 & -1.16809628753955 \\
\hline $\mathrm{N}$ & -0.93201539368408 & 1.35460123070186 & -0.35560380236429 \\
\hline $\mathrm{C}$ & 0.55190843365427 & 1.38628663281294 & -0.60145363496436 \\
\hline $\mathrm{C}$ & 1.19656704020148 & 2.76394618085713 & -0.48747320385860 \\
\hline $\mathrm{N}$ & 2.63186208081176 & 2.58721572344591 & -0.56476499307986 \\
\hline $\mathrm{C}$ & 3.38704816641135 & 3.76008870180766 & -0.13825252727466 \\
\hline $\mathrm{C}$ & -1.46874244054830 & -0.02091947979713 & -0.61600216655639 \\
\hline $\mathrm{C}$ & -1.02674960598858 & -1.02012558375736 & 0.45109029346893 \\
\hline $\mathrm{N}$ & -1.80283957778230 & -2.21655062228576 & 0.33345862915796 \\
\hline $\mathrm{H}$ & -3.37627124368584 & 3.35734418590255 & -3.12296368335366 \\
\hline $\mathrm{H}$ & -2.22717240211999 & 3.28489363648811 & -4.45831966330342 \\
\hline
\end{tabular}




\begin{tabular}{|c|c|c|c|}
\hline $\mathrm{H}$ & -1.91628600984889 & 4.35604181918988 & -3.09239858503024 \\
\hline $\mathrm{H}$ & -0.49971506487929 & 2.28400673841073 & -2.97212320107603 \\
\hline $\mathrm{H}$ & -1.94633400404152 & 1.28312666525074 & -3.01517058678975 \\
\hline $\mathrm{H}$ & -2.74015307640882 & 2.28786925030098 & -0.87075196075817 \\
\hline $\mathrm{H}$ & -1.34675228256046 & 3.36491533365448 & -0.84741935464284 \\
\hline $\mathrm{H}$ & 0.72996500793435 & 0.95237435121575 & -1.59024878310611 \\
\hline $\mathrm{H}$ & 1.03220107024053 & 0.74099403536496 & 0.13571456336334 \\
\hline $\mathrm{H}$ & 0.96882783470120 & 3.19124763261359 & 0.49580338779760 \\
\hline $\mathrm{H}$ & 0.80356321918079 & 3.45683738337427 & -1.24954916399157 \\
\hline $\mathrm{H}$ & 2.90401404008656 & 2.32926240079926 & -1.50616066626242 \\
\hline $\mathrm{H}$ & 3.12924813284134 & 4.67688522426827 & -0.68932977041568 \\
\hline $\mathrm{H}$ & 4.45232222015434 & 3.56728150273679 & -0.26903737755988 \\
\hline $\mathrm{H}$ & 3.21087535124835 & 3.93619529496423 & 0.92589071682213 \\
\hline $\mathrm{H}$ & -1.14879608544805 & -0.36358592852166 & -1.60105725718843 \\
\hline $\mathrm{H}$ & -2.55938111055906 & 0.05297151189050 & -0.61146796547826 \\
\hline $\mathrm{H}$ & -1.10506936173637 & -0.53738304600568 & 1.46940562061337 \\
\hline
\end{tabular}




\begin{tabular}{|c|c|c|c|}
\hline $\mathrm{H}$ & 0.03064557769635 & -1.26937402626267 & 0.34092911820838 \\
\hline $\mathrm{H}$ & -2.76881605012969 & -2.10576540333190 & 0.61217981767858 \\
\hline $\mathrm{H}$ & -1.39864370329678 & -3.00834163768755 & 0.81248757763149 \\
\hline $\mathrm{Ga}$ & -1.35840862159975 & 1.66189479945666 & 1.54277172463223 \\
\hline $\mathrm{O}$ & -3.11271418202859 & 1.55527746649052 & 1.65891038981421 \\
\hline $\mathrm{O}$ & 0.02114066556500 & 2.12504973100016 & 2.52319916136647 \\
\hline $\mathrm{H}$ & -3.56235748578550 & 1.76316256118386 & 2.47993390317410 \\
\hline $\mathrm{H}$ & -0.09522515265717 & 2.30008914314282 & 3.45942619407237 \\
\hline \multicolumn{4}{|c|}{$\mathrm{PEI}^{\cdots} \mathrm{NH}-\mathrm{Ga}(\mathrm{OH})_{2}{ }^{+}$} \\
\hline $\mathrm{C}$ & -2.91038481825096 & 2.44711396596672 & -4.00853948289376 \\
\hline $\mathrm{C}$ & -1.91184598028604 & 1.76384769120514 & -3.07807247629495 \\
\hline $\mathrm{C}$ & -1.95928313612277 & 2.35576233792044 & -1.66784147941187 \\
\hline $\mathrm{N}$ & -1.01519835864396 & 1.78842070497940 & -0.69767049786074 \\
\hline $\mathrm{C}$ & 0.35291278204103 & 2.12585726070894 & -0.94616049701151 \\
\hline $\mathrm{C}$ & 1.08094020505065 & 2.64386796519342 & 0.29448888636678 \\
\hline $\mathrm{N}$ & 2.55231429413320 & 2.70334939736514 & 0.02383667200035 \\
\hline
\end{tabular}




\begin{tabular}{|c|c|c|c|}
\hline $\mathrm{C}$ & 3.34525318619131 & 3.02407471257986 & 1.24643938047609 \\
\hline $\mathrm{C}$ & -1.25068436107961 & 0.37627375725637 & -0.40193544807363 \\
\hline $\mathrm{C}$ & -0.74573232702113 & -0.08289527505740 & 0.96368391194032 \\
\hline $\mathrm{N}$ & -1.07794504395205 & -1.48918531171806 & 1.13499111624448 \\
\hline $\mathrm{H}$ & -3.93346665130919 & 2.33626609836103 & -3.63802266241212 \\
\hline $\mathrm{H}$ & -2.87381597951102 & 2.01783290608830 & -5.01144719699284 \\
\hline $\mathrm{H}$ & -2.70397944978414 & 3.51790228839418 & -4.09776780961948 \\
\hline $\mathrm{H}$ & -0.89911274388811 & 1.86261302638817 & -3.49105368679914 \\
\hline $\mathrm{H}$ & -2.12510552289109 & 0.69049775310591 & -3.03487857283175 \\
\hline $\mathrm{H}$ & -2.96245580481603 & 2.23411027536773 & -1.24792830787847 \\
\hline $\mathrm{H}$ & -1.78231073347081 & 3.43648459441317 & -1.71122543216575 \\
\hline $\mathrm{H}$ & 0.42471135211195 & 2.87474432220180 & -1.74280961642780 \\
\hline $\mathrm{H}$ & 0.90314052086841 & 1.22554562952762 & -1.34990903216007 \\
\hline $\mathrm{H}$ & 0.93338315256861 & 1.97823585614160 & 1.14503420218258 \\
\hline $\mathrm{H}$ & 0.71938071043274 & 3.63745674251853 & 0.56758189223666 \\
\hline $\mathrm{H}$ & 2.72205359775310 & 3.43555742496365 & -0.66667495131429 \\
\hline
\end{tabular}




\begin{tabular}{|c|c|c|c|}
\hline $\mathrm{H}$ & 2.97346441384969 & 3.94654054793414 & 1.69438484180908 \\
\hline $\mathrm{H}$ & 4.39487921370501 & 3.14982434995762 & 0.97898086440803 \\
\hline $\mathrm{H}$ & 3.24376370782636 & 2.20350078415590 & 1.95667320304769 \\
\hline $\mathrm{H}$ & -0.81109952173399 & -0.28095896639476 & -1.17116700451445 \\
\hline $\mathrm{H}$ & -2.33252727923281 & 0.21758402103460 & -0.43706602663995 \\
\hline $\mathrm{H}$ & -1.15824098009340 & 0.58550572404246 & 1.73567610122102 \\
\hline $\mathrm{H}$ & 0.34638727682766 & -0.01125454931412 & 1.00912395175356 \\
\hline $\mathrm{H}$ & -2.07801467226697 & -1.62623080784788 & 1.22482683133908 \\
\hline $\mathrm{H}$ & -0.64842654543734 & -1.87301579883423 & 1.96658817212824 \\
\hline $\mathrm{Ga}$ & 3.05449056525871 & 0.99310812719433 & -0.82297396365246 \\
\hline $\mathrm{O}$ & 2.84726467746065 & -0.22427841556182 & 0.42212787328069 \\
\hline $\mathrm{O}$ & 3.76062641542432 & 1.34364210621088 & -2.38587586438505 \\
\hline $\mathrm{H}$ & 3.00219522669403 & -1.15256514975719 & 0.23452714675860 \\
\hline $\mathrm{H}$ & 4.12915561159395 & 0.64618690330822 & -2.93211903785313 \\
\hline \multicolumn{4}{|c|}{$\mathrm{PEI} \cdots \mathrm{NH}_{2}-\mathrm{Ga}(\mathrm{OH})_{2}{ }^{+}$} \\
\hline $\mathrm{C}$ & -2.90560781084134 & 2.75776858494591 & -3.81607924333653 \\
\hline
\end{tabular}




\begin{tabular}{|c|c|c|c|}
\hline $\mathrm{C}$ & -1.91277943006456 & 1.88136261223244 & -3.05751243065178 \\
\hline $\mathrm{C}$ & -1.80949808816165 & 2.28245786151991 & -1.58436982775365 \\
\hline $\mathrm{N}$ & -0.82431249088293 & 1.53496233797802 & -0.80087174111349 \\
\hline $\mathrm{C}$ & 0.57673286357446 & 1.90123081785659 & -1.01494118256129 \\
\hline $\mathrm{C}$ & 1.15539060481018 & 2.69951666199559 & 0.14886864169113 \\
\hline $\mathrm{N}$ & 2.56103621221478 & 2.96411560573281 & -0.07578722976005 \\
\hline $\mathrm{C}$ & 3.21048779782171 & 3.63527170900141 & 1.04241657780512 \\
\hline $\mathrm{C}$ & -1.09237231328418 & 0.16337757039248 & -0.63501805445756 \\
\hline $\mathrm{C}$ & -0.43657398422887 & -0.45389006416497 & 0.59667269318072 \\
\hline $\mathrm{N}$ & -0.58487770165395 & -1.94164226177409 & 0.50664724992576 \\
\hline $\mathrm{H}$ & -3.90746130343212 & 2.69015359636229 & -3.38230142324940 \\
\hline $\mathrm{H}$ & -2.97701772100501 & 2.45790808245791 & -4.86317799881373 \\
\hline $\mathrm{H}$ & -2.60278468731422 & 3.80838049046590 & -3.79234280970212 \\
\hline $\mathrm{H}$ & -0.92494245834445 & 1.94832391657122 & -3.52850366494779 \\
\hline $\mathrm{H}$ & -2.22469687481323 & 0.83141817818465 & -3.13265512624780 \\
\hline $\mathrm{H}$ & -2.78525812486555 & 2.17261339390736 & -1.09786194675961 \\
\hline
\end{tabular}




\begin{tabular}{|c|c|c|c|}
\hline $\mathrm{H}$ & -1.54856949819789 & 3.34165216299869 & -1.50140287220484 \\
\hline $\mathrm{H}$ & 0.66004605096286 & 2.48775721321904 & -1.93689740242881 \\
\hline $\mathrm{H}$ & 1.19268826540444 & 1.00764963302530 & -1.17725145065320 \\
\hline $\mathrm{H}$ & 1.06201035657964 & 2.11444588242312 & 1.07204584562249 \\
\hline $\mathrm{H}$ & 0.54801572022464 & 3.60853383116350 & 0.30306838677527 \\
\hline $\mathrm{H}$ & 2.67988292373057 & 3.51623282546539 & -0.91731190813048 \\
\hline $\mathrm{H}$ & 2.71951352356216 & 4.57481774025637 & 1.33863271011900 \\
\hline $\mathrm{H}$ & 4.24760252849846 & 3.85353852023092 & 0.78405495897852 \\
\hline $\mathrm{H}$ & 3.22040468509354 & 2.97095316686893 & 1.91102268607757 \\
\hline $\mathrm{H}$ & -0.72553322467053 & -0.44876482127658 & -1.54509688970827 \\
\hline $\mathrm{H}$ & -2.17441977492807 & -0.00586891246372 & -0.61606647759988 \\
\hline $\mathrm{H}$ & -0.89277508281122 & -0.07844125744328 & 1.51316385387860 \\
\hline $\mathrm{H}$ & 0.63248097562420 & -0.23692092414344 & 0.61722158609236 \\
\hline $\mathrm{H}$ & -1.52358512021193 & -2.22924873941238 & 0.78302060191667 \\
\hline $\mathrm{H}$ & 0.06361919594786 & -2.40215881523537 & 1.14284519240845 \\
\hline $\mathrm{Ga}$ & -0.27412993919575 & -2.45848726427114 & -1.39088853779963 \\
\hline
\end{tabular}




\begin{tabular}{|l|l|l|l|}
\hline $\mathrm{O}$ & 1.46438000464362 & -2.38651404002266 & -1.61172128774762 \\
\hline $\mathrm{O}$ & -1.78132525405808 & -3.15686288953469 & -1.95514535943298 \\
\hline $\mathrm{H}$ & 1.87232962699868 & -2.65048239193517 & -2.43869030674160 \\
\hline $\mathrm{H}$ & -1.84174045272627 & -3.58825001357823 & -2.80968581266954 \\
& & & \\
\hline
\end{tabular}

Table S3. Comparison of the different machine learning models accuracy.

\begin{tabular}{|l|l|}
\hline ML Model & Correctly classified instances, $\%$ \\
\hline Naive Bayes & $42 \%$ \\
\hline Logistic regression & $30 \%$ \\
\hline Simple Logistic regression & $29 \%$ \\
\hline Random Tree & $77 \%$ \\
\hline Multilayer Perceptron & \\
\hline EM Clusterers & $82 \%$ \\
\hline SimpleKMeans Clusterers & $22 \%$ \\
\hline & $18 \%$ \\
\hline
\end{tabular}


Table S4. Extracted light intensities from diodes corresponds the conductivity of the hydrogel/eGaIn interface depicted in Figure 5.

\begin{tabular}{|c|c|c|c|c|c|c|}
\hline & \multicolumn{3}{|c|}{ input } & \multicolumn{3}{|c|}{ output } \\
\hline & $\begin{array}{c}\text { PEI in } \\
\text { PBS } 4.8\end{array}$ & $\begin{array}{l}\text { PAA in } \\
\text { PBS } 4.8\end{array}$ & $\begin{array}{l}\text { PBS } \\
4.8\end{array}$ & $\begin{array}{r}\text { PEI in } \\
\text { PBS } 4.8\end{array}$ & $\begin{array}{l}\text { PAA in } \\
\text { PBS } 4.8\end{array}$ & PBS 4.8 \\
\hline$-3 \mathrm{~V}$ & 1990 & 740 & 520 & 1496 & 1640 & 912 \\
\hline $3 \mathrm{~V}$ & 3874 & 3120 & 510 & 814 & 4572 & 1976 \\
\hline$-4 \mathrm{~V}$ & 25190 & 8150 & 3970 & 13794 & 17080 & 11700 \\
\hline $4 \mathrm{~V}$ & 33618 & 27264 & 7600 & 10934 & 36276 & 24349 \\
\hline$-5 \mathrm{~V}$ & 29940 & 22880 & 16940 & 35134 & 33610 & 27348 \\
\hline $5 \mathrm{~V}$ & 36868 & 31092 & 20800 & 25333 & 43536 & 36868 \\
\hline$-6 \mathrm{~V}$ & 29670 & 26640 & 24150 & 32615 & 31190 & 36264 \\
\hline $6 \mathrm{~V}$ & 33319 & 27912 & 21350 & 25762 & 41868 & 34515 \\
\hline
\end{tabular}




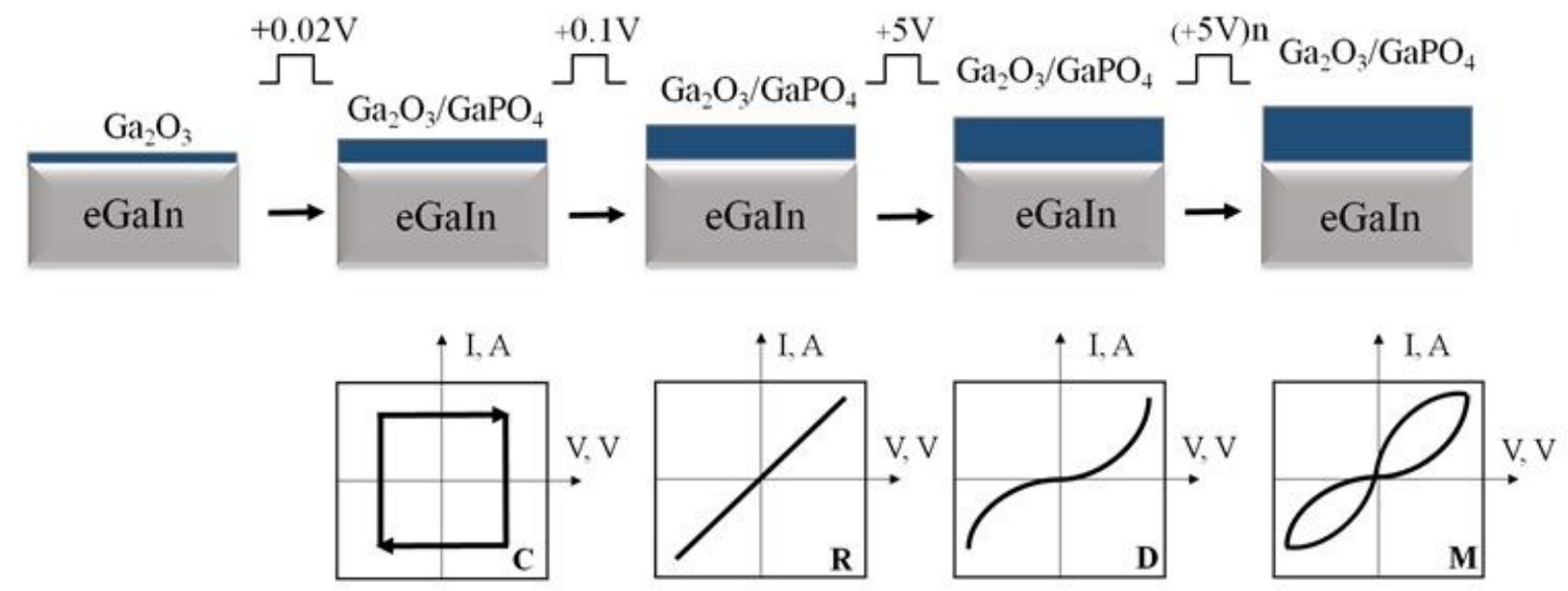

Figure S1. Dependence of electrical behavior from thickness of oxide-phosphate layer. The formation of a film leads to a change in the electrical behavior of the system in the order: capacitor, resistor, diode, memristor.

a)

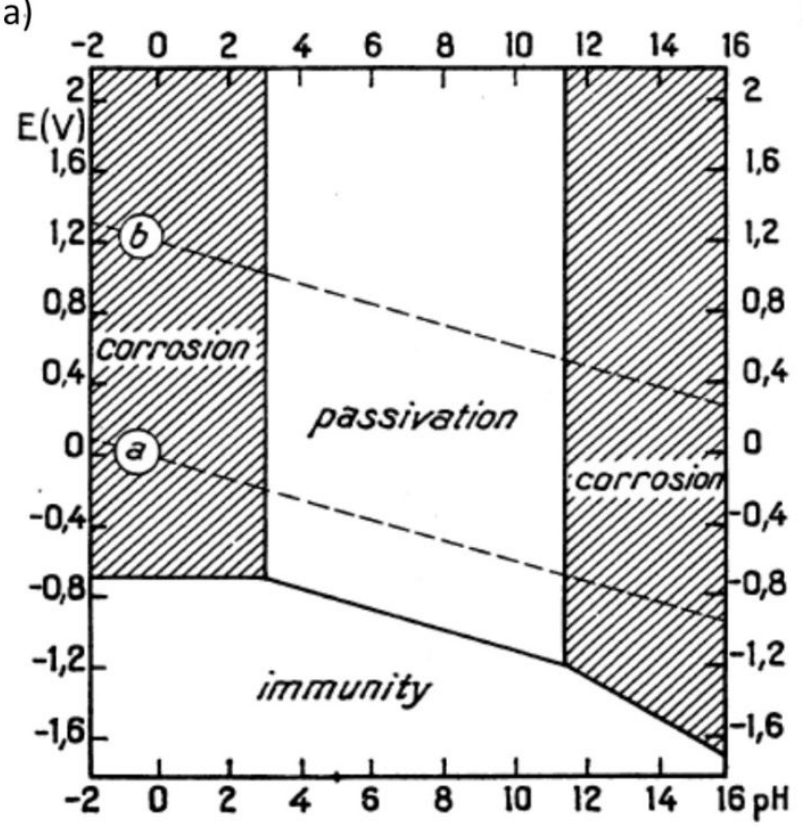

b)

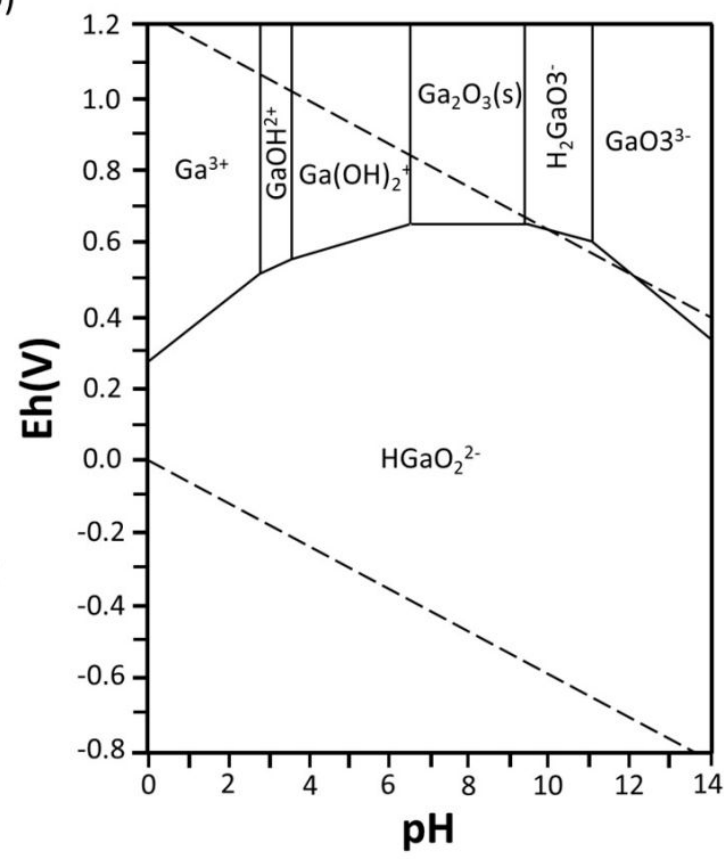

Figure S2. Pourbaix atlas data ${ }^{2}$ of the theoretical conditions for: a) $\alpha-\mathrm{Ga}_{2} \mathrm{O}_{3}$ film corrosion, immunity and passivation at $25^{\circ} \mathrm{C}$, b) Standard redox potentials for the gallium transition forms depends on applied voltage and $\mathrm{pH}$. 


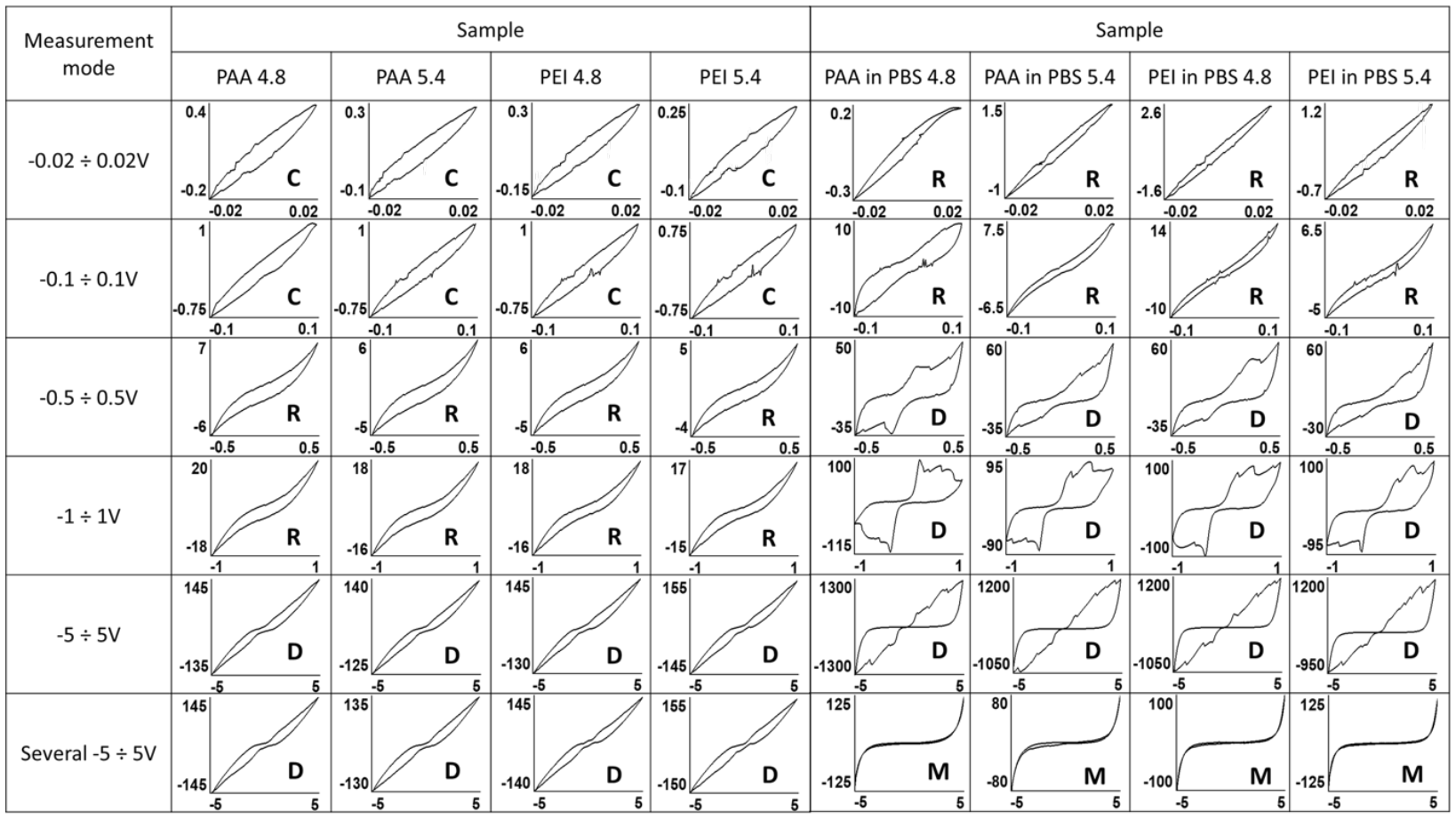

Figure S3. I-V curves used for a database showing electrical behaviors (capacitor (C), resistor (R), diode (D), memristor (M)) depending on gel composition. 
a)

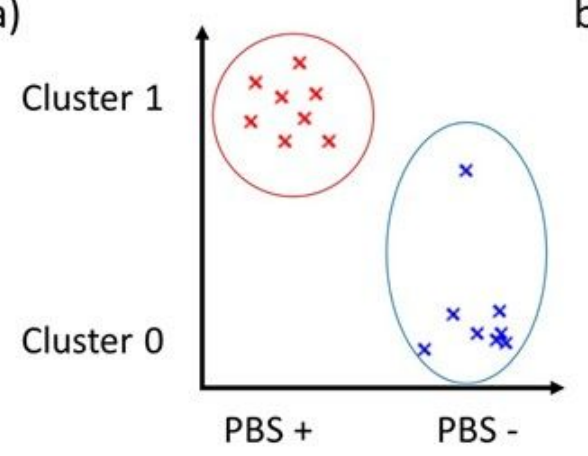

c)

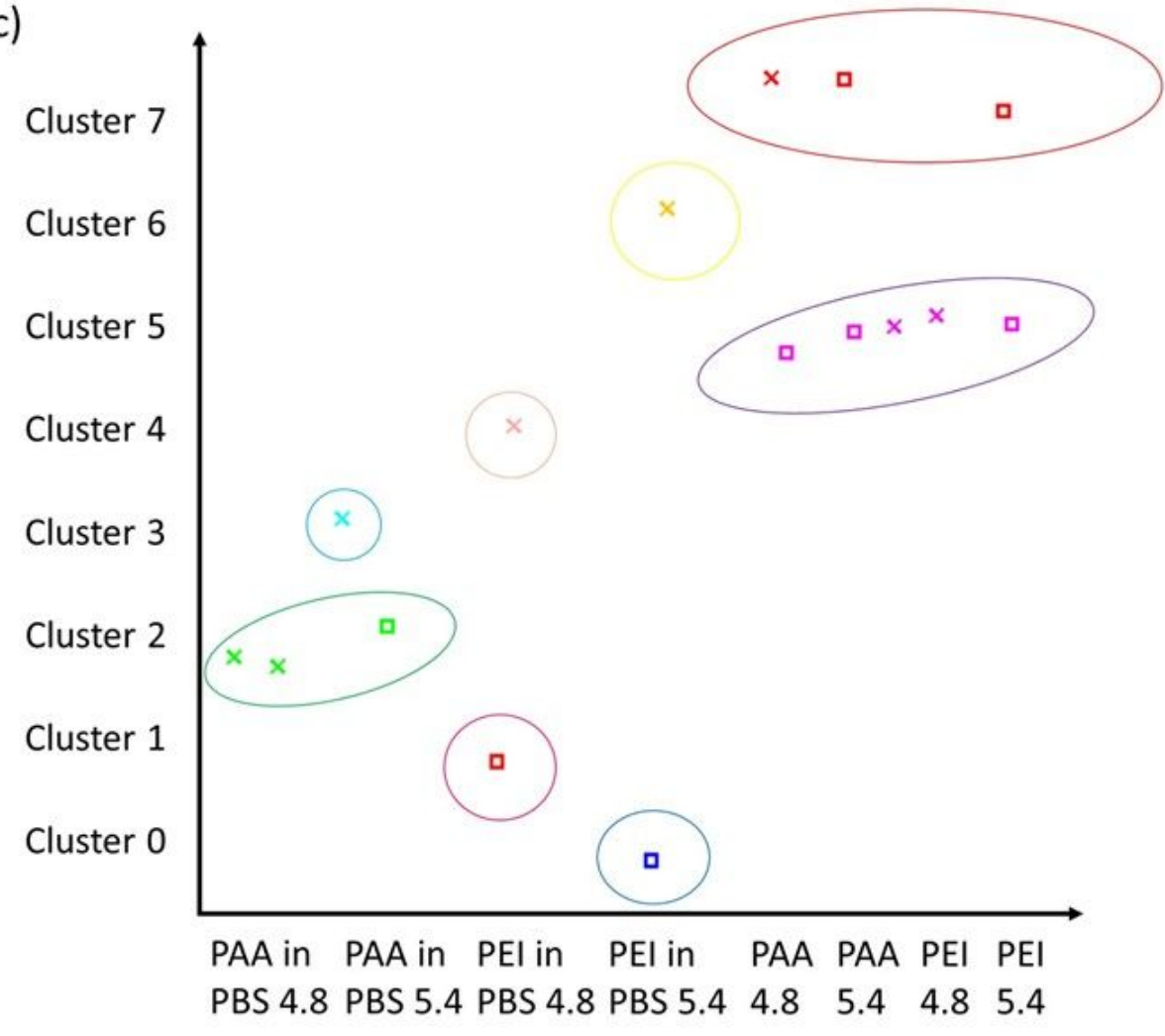

Figure S4. Results of unsupervised learning by the expectation maximization (EM) clustering model. a) Determining the presence of a buffer is performed with $100 \%$ accuracy. b) The polyelectrolyte is determined with an accuracy of $50 \%$. c) The determination of all system parameters is determined with $50 \%$ accuracy. 

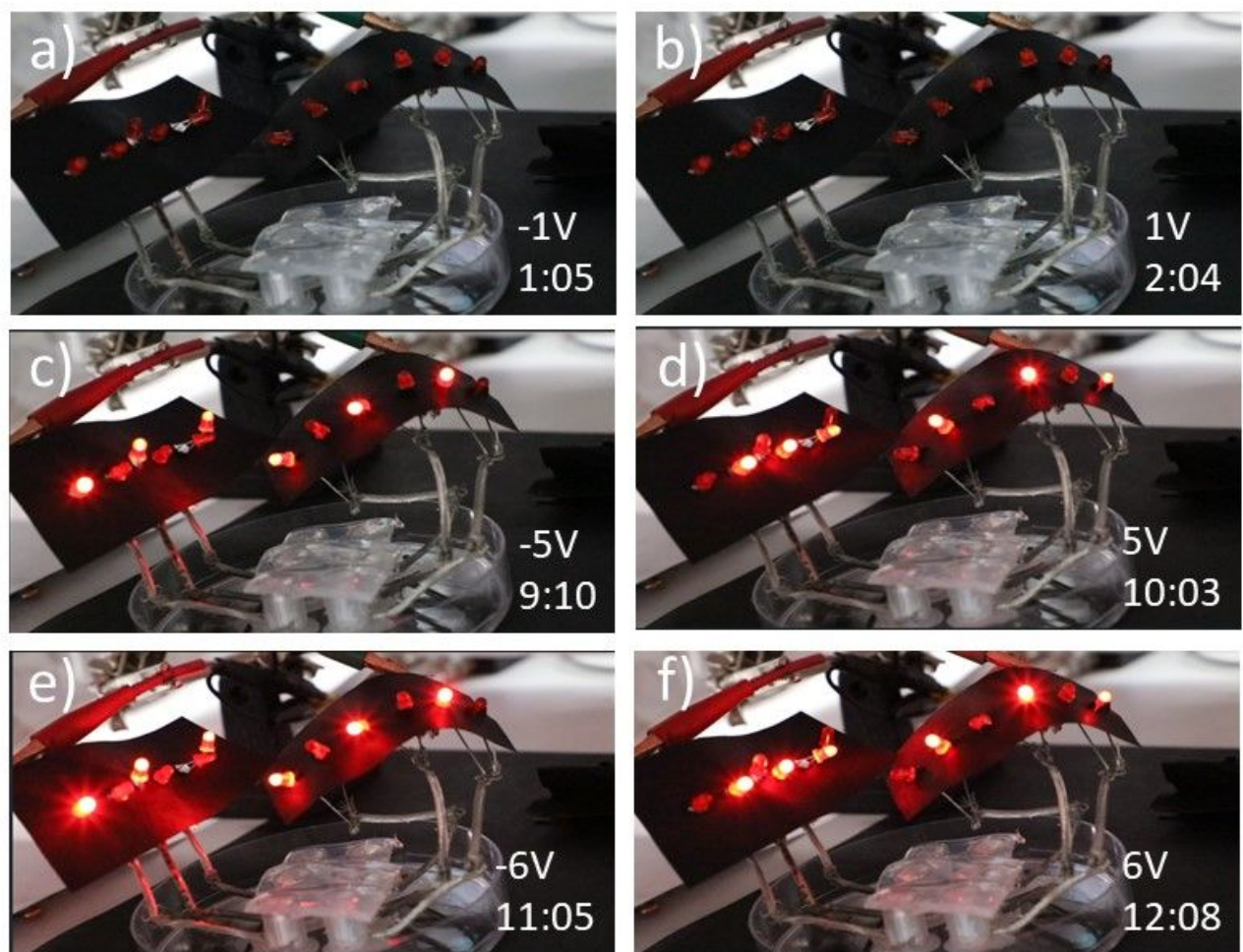

Figure S5. a) Set of experiments with different values of applied voltage -1V forward current direction, b) $1 \mathrm{~V}$ reverse current direction, c) $-5 \mathrm{~V}$ forward current direction, d) $5 \mathrm{~V}$ reverse current direction, e) $-6 \mathrm{~V}$ forward current direction, f) $6 \mathrm{~V}$ reverse current direction. Intensity of the diode's brightness depends on the film resistance.

See also attached Video S1.

(1) Neese, F. The ORCA program system. WIREs Computational Molecular Science 2012, 2 (1), 73-78.;

(2) Pourbaix, M. Atlas of electrochemical equilibria in aqueous solution. NACE 1974, 307. 